\title{
Biomechanical study of a hat type cervical intervertebral fusion cage
}

\author{
Yu-Tong Gu $\cdot$ Lian-Shun Jia $\cdot$ Tong-Yi Chen
}

Published online: 20 October 2006

(C) Springer-Verlag 2006

The online version of the original article can be found at http://dx.doi. org/10.1007/s00264-006-0141-8.

Y.-T. Gu $(\bowtie) \cdot$ T.-Y. Chen

Department of Orthopaedics,

Zhongshan Hospital of Fudan University,

Shanghai 200032, People's Republic of China

e-mail: gyt633@sohu.com

L.-S. Jia

Department of Orthopaedics, Changzheng Hospital

of Shanghai Second Military Medical University,

Shanghai 200003, People's Republic of China
The authors' addresses were given wrongly in the original article. The correct wording is given here. 